\title{
Temporal Dynamics of Photon Pairs Generated by an Atomic Ensemble
}

\author{
S. V. Polyakov, C. W. Chou, D. Felinto, and H. J. Kimble \\ Norman Bridge Laboratory of Physics 12-33, California Institute of Technology, Pasadena, California 91125, USA
}

(Received 4 June 2004; published 20 December 2004)

\begin{abstract}
The time dependence of nonclassical correlations is investigated for two fields $(1,2)$ generated by an ensemble of cold cesium atoms via the protocol of Duan et al. [Nature (London) 414, 413 (2001)]. The correlation function $R\left(t_{1}, t_{2}\right)$ for the ratio of cross to autocorrelations for the $(1,2)$ fields at times $\left(t_{1}, t_{2}\right)$ is found to have a maximum value $R^{\max }=292 \pm 57$, which significantly violates the Cauchy-Schwarz inequality $R \leq 1$ for classical fields. Decoherence of quantum correlations is observed over $\tau_{d} \simeq 175 \mathrm{ns,}$ and is described by our model, as is a new scheme to mitigate this effect.
\end{abstract}

DOI: 10.1103/PhysRevLett.93.263601

PACS numbers: 42.50.Dv, 03.67. $-\mathrm{a}, 42.65 .-\mathrm{k}$

In recent years quantum measurement combined with conditional quantum evolution has emerged as a powerful paradigm for accomplishing diverse tasks in quantum information science [1-4]. For example, Duan, Lukin, Cirac, and Zoller (DLCZ) [4] have proposed a scheme for scalable quantum communication networks that relies upon a probabilistic entanglement between remotely located atomic ensembles. By utilizing successful measurements to condition subsequent steps in the protocol, DLCZ have developed a scheme that has built-in quantum memory, entanglement purification, and resilience to realistic noise sources, enabling a quantum repeater architecture to overcome photon attenuation $[5,6]$.

Central to the DLCZ protocol is the ability to write and read collective spin excitations into and out of an atomic ensemble, with efficient conversion of discrete spin excitations to single-photon wave packets. Observations of the resulting nonclassical correlations between the optical fields generated from writing and reading such spin excitations have recently been reported both at the singlephoton level [7-11] as appropriate to the protocol of DLCZ and in a regime of large photon number $n \sim 10^{3}-10^{7}$ [12]. Generation and detection efficiencies have now been improved so that excitation stored within an atomic ensemble can be employed as a controllable source for single photons [8].

A critical aspect of such single-photon wave packets is that they are emitted into well defined spatiotemporal modes to enable quantum interference between emissions from separate ensembles (e.g., for entanglement based quantum cryptography [4]). However, with the exception of the verification of the time delay implicit for the Raman processes employed [12], experiments to date have not investigated the detailed time and spatial dependence of quantum correlations for the emitted fields from the atomic ensemble. The high efficiencies achieved in Ref. [8] now enable such an investigation for the temporal properties of nonclassical correlations between emitted photon pairs, which we report in this Letter.

We study the time dependence of quantum correlations for photons emitted from an ensemble of cold cesium atoms, with photon pairs created by classically controlled write and read pulses. We also map the decay of quantum correlations by varying the delay between the write and read pulses. We have developed a model to describe the decoherence and find good correspondence with our measurements. This model is utilized to analyze a new proposal that should extend the correlation times to beyond $10 \mu \mathrm{s}$, which would allow for entanglement between atomic ensembles on the scale of several kilometers.

The DLCZ scheme is implemented in an optically thick sample of four-level atoms, cooled and trapped in a magneto-optical trap (MOT). In particular, we utilize the ground hyperfine levels $6 S_{1 / 2}, F=\{4 ; 3\}$ of Cs (labeled $\{|a\rangle ;|b\rangle\})$, and excited levels $\left\{6 P_{3 / 2}, F=4 ; 6 P_{1 / 2}, F=4\right\}$ of the $D_{2}, D_{1}$ lines (labeled $\left\{|e\rangle ;\left|e^{\prime}\right\rangle\right\}$ ). Our experimental procedure is illustrated in Fig. 1. An initial write pulse at $852 \mathrm{~nm}$ creates a state of collective excitation in an atomic

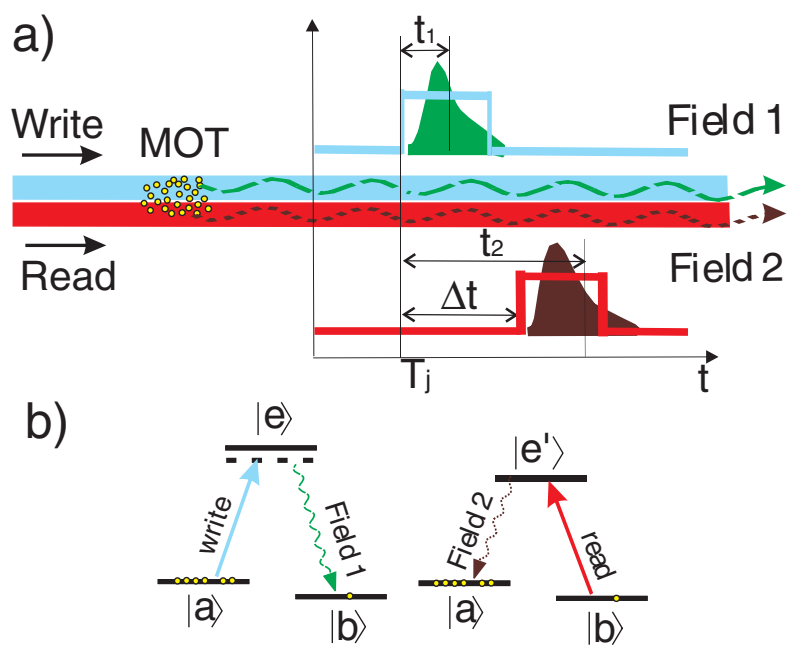

FIG. 1 (color online). (a) Schematic of experiment. Write and read pulses propagate into a cloud of cold Cs atoms (MOT) at times $T_{j}$ and $T_{j}+\Delta t$, respectively, and generate the correlated output fields 1 and 2. Quantum correlations for these fields at times $\left(t_{1}, t_{2}\right)$ are investigated by way of photoelectric detection. (b) The relevant atomic level scheme. 
ensemble, as heralded by a photoelectric detection from the Raman field 1. After a user-programmable delay $\Delta t$, a read pulse at $894 \mathrm{~nm}$ converts this atomic excitation into a field excitation of the Raman field 2. The (write, read) pulses are approximate coherent states with mean photon numbers $\left(10^{3}, 10^{5}\right)$, respectively, focused into MOT as Gaussian beams with orthogonal polarizations and beam waist $w_{0}=30 \mu \mathrm{m}$.

Each attempt to generate a correlated pair of photons in the $(1,2)$ fields is preceded by shutting off the trapping light for $700 \mathrm{~ns}$. The repumping light is left on for an additional $300 \mathrm{~ns}$ in order to empty the $|b\rangle$ state, thus preparing the atoms in $|a\rangle$. The $j$ th trial of a protocol is initiated at time $T_{j}$ when a rectangular pulse from the write laser beam, $150 \mathrm{~ns}$ in duration and tuned $10 \mathrm{MHz}$ below the $|a\rangle \rightarrow|e\rangle$ transition, induces spontaneous Raman scattering to level $|b\rangle$ via $|a\rangle \rightarrow|e\rangle \rightarrow|b\rangle$. The write pulse is weak so that the probability to scatter one Raman photon into a forward propagating wave packet is much less than unity for each pulse. Detection of one photon from field 1 results in a "spin" excitation to level $|b\rangle$, with this excitation distributed in a symmetrized, coherent manner throughout the sample of $N$ atoms illuminated by the write beam [4]. Regardless of detection of a photon in field 1, we next address the ensemble with a read pulse at a time $T_{j}+$ $\Delta t$, where $\Delta t$ is controlled by the user. The read light is a rectangular pulse, $120 \mathrm{~ns}$ in duration, resonant with the $|b\rangle \rightarrow\left|e^{\prime}\right\rangle$ transition.

To address the photon statistics, we use four avalanche photodetectors, a pair for each field $(1,2)$, activated at $\left(T_{j}, T_{j}+\Delta t\right)$, respectively, for $200 \mathrm{~ns}$ for all experiments. We define $p_{\tau}\left(t_{l}, t_{m}\right)$ as the joint probability for photoelectric detection from field $l$ in the interval $\left[T_{j}+t_{l}, T_{j}+t_{l}+\right.$ $\tau]$ and for detection from field $m$ in the interval $\left[T_{j}+\right.$ $\left.t_{m}, T_{j}+t_{m}+\tau\right]$ in a temporal bin $\tau ; l$ and $m$ equal 1 or 2 . $p_{\tau}\left(t_{l}, t_{m}\right)$ is determined from the record of time-stamped detection events of all four photodetectors, allowing us to measure autocorrelations and cross correlations simultaneously. Similarly, $q_{\tau}\left(t_{l}, t_{m}\right)$ gives the joint probability for detection for fields $(l, m)$ in the intervals $\left(\left[T_{j}+t_{l}, T_{j}+\right.\right.$ $\left.\left.t_{l}+\tau\right],\left[T_{k}+t_{m}, T_{k}+t_{m}+\tau\right]\right)$ for two trials $k \neq j$.

Following Refs. [7,8], we introduce the time-dependent ratio $R_{\tau}\left(t_{1}, t_{2}\right)$ of cross correlation to autocorrelation for the $(1,2)$ fields, where

$$
R_{\tau}\left(t_{1}, t_{2}\right) \equiv \frac{\left[p_{\tau}\left(t_{1}, t_{2}\right)\right]^{2}}{p_{\tau}\left(t_{1}, t_{1}\right) p_{\tau}\left(t_{2}, t_{2}\right)}
$$

This ratio is constrained by the inequality $R_{\tau}\left(t_{1}, t_{2}\right) \leq 1$ for all fields for which the Glauber-Sudarshan phase-space function is well behaved (i.e., classical fields) [7,13]. Beyond enabling a characterization of the quantum character of the $(1,2)$ fields in a model independent fashion, measurements of $R_{\tau}\left(t_{1}, t_{2}\right)$ also allow inferences of the quantum state for collective excitations of single spins within the atomic ensemble.
The first step in the determination of $R_{\tau}\left(t_{1}, t_{2}\right)$ is the measurement of the joint probability $p_{\tau}\left(t_{1}, t_{2}\right)$ for the $(1,2)$ fields, and, for comparison, $q_{\tau}\left(t_{1}, t_{2}\right)$ for independent trials. In our experiment, we focus on two cases: (I) nearly simultaneous application of write and read pulses with offset $\Delta t=50 \mathrm{~ns}$ less than the duration of either pulse, and (II) consecutive application of write and read pulses with $\Delta t=$ $200 \mathrm{~ns}$ longer than the write, read durations. Results for $p_{\tau}\left(t_{1}, t_{2}\right)$ and $q_{\tau}\left(t_{1}, t_{2}\right)$ are presented in Fig. 2 as functions of the detection times $\left(t_{1}, t_{2}\right)$ for the fields $(1,2)$. For both $\Delta t=50$ and $200 \mathrm{~ns}, p_{\tau}\left(t_{1}, t_{2}\right) \gg q_{\tau}\left(t_{1}, t_{2}\right)$, indicating the strong correlation between fields 1 and 2 , with the maximal ratio $g_{1,2}^{\tau}\left(t_{1}, t_{2}\right)=p_{\tau}\left(t_{1}, t_{2}\right) / q_{\tau}\left(t_{1}, t_{2}\right) \gtrsim 30$, much greater than reported previously [7-9]. In Fig. 2, $\tau=4$ ns, leading to statistical errors of $\approx 8 \%$ for the largest values shown.

In case (I) for nearly simultaneous write and read pulses, Fig. 2(a) shows that $p_{\tau}\left(t_{1}, t_{2}\right)$ peaks along the line $t_{2}-$ $t_{1}=\delta t_{12} \simeq 50 \mathrm{~ns}$ with a width $\Delta t_{2} \simeq 60 \mathrm{~ns}$, in correspondence to the delay $\delta t_{12}$ and duration $\Delta t_{2}$ for readout associated with the transition $|b\rangle \rightarrow\left|e^{\prime}\right\rangle \rightarrow|a\rangle$ given an initial transition $|a\rangle \rightarrow|e\rangle \rightarrow|b\rangle$ [12]. Apparently, the qualitative features of $p_{\tau}\left(t_{1}, t_{2}\right)$ depend only upon the time difference between photon detections in fields $(1,2)$ [i.e., $p_{\tau}\left(t_{1}, t_{2}\right) \approx F\left(t_{2}-t_{1}\right)$ ]. In case (II) with the read pulse launched $200 \mathrm{~ns}$ after the write pulse, excitation is "stored" in the atomic ensemble until the readout. The production of correlated photon pairs should now be distributed along $t_{2} \simeq \Delta t+\delta t_{12}$ with width $\simeq \Delta t_{2}$. Instead, as shown in Fig. 2(c), $p_{\tau}\left(t_{1}, t_{2}\right)$ peaks towards the end of the write pulse (i.e., $t_{1} \gtrsim 100 \mathrm{~ns}$ ), and near the beginning of the read pulse (i.e., $200 \leqq t_{2} \lesssim 300 \mathrm{~ns}$ ). Early events for field 1 lead to fewer correlated events for field 2 , as
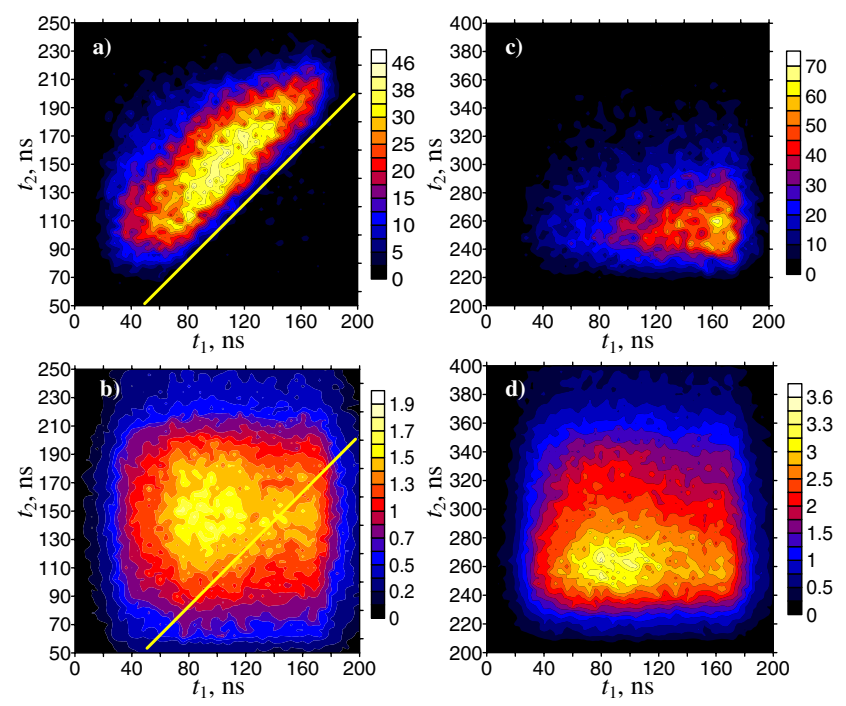

FIG. 2 (color online). Probability of joint detection scaled by $10^{9}$ from fields $(1,2)$ at times $\left(t_{1}, t_{2}\right)$ with $t_{1}=t_{2}=0$ at the beginning of the write pulse. (a) $p_{\tau}\left(t_{1}, t_{2}\right)$ and (b) $q_{\tau}\left(t_{1}, t_{2}\right)$ for overlapped write and read pulses, $\Delta t=50 \mathrm{~ns}$. The solid line corresponds to $t_{2}=t_{1}$. (c) $p_{\tau}\left(t_{1}, t_{2}\right)$ and (d) $q_{\tau}\left(t_{1}, t_{2}\right)$ for consecutive write and read pulses, $\Delta t=200 \mathrm{~ns}$. 
$p_{\tau}\left(t_{1}, t_{2}\right)$ decays rapidly beyond $t_{2}-t_{1}=\tau_{d} \simeq 175 \mathrm{~ns}$. The marked contrast between $p_{\tau}\left(t_{1}, t_{2}\right)$ for $\Delta t=50$ and $200 \mathrm{~ns}$ results in a diminished ability for the conditional generation of single photons from excitation stored within the atomic ensemble [8] and for the implementation of the DLCZ protocol for increasing $\Delta t$. The underlying mechanism is Zeeman decoherence, as discussed below.

Figures 2(b) and 2(d) displays $q_{\tau}\left(t_{1}, t_{2}\right)$ for independent trials $j \neq k . q_{\tau}\left(t_{1}, t_{2}\right)$ is expected to be proportional to the product of intensities of the fields 1 and 2 , in reasonable correspondence to the form shown in Figs. 2(b) and 2(d) for our roughly rectangular write, read pulses, but distinctively different from $p_{\tau}\left(t_{1}, t_{2}\right)$ in Figs. 2(a) and 2(c).

To deduce $R_{\tau}\left(t_{1}, t_{2}\right)$ from Eq. (1), we determine the joint detection probabilities $p_{\tau}\left(t_{i}, t_{i}\right)$ for fields 1 and 2 from the same record of photoelectric events. Since the rate of coincidences for autocorrelations is roughly $10^{2}$ times smaller than for cross correlations for the $(1,2)$ fields, we increase the bin size $\tau$ to $30 \mathrm{~ns}$ to accumulate enough events to reduce the statistical errors to acceptable levels. Figure 3 shows the time dependencies of $p_{\tau}\left(t_{1}, t_{1}\right)$ and $p_{\tau}\left(t_{2}, t_{2}\right)$ for cases (I) and (II). While the shape of $p_{\tau}\left(t_{1}, t_{1}\right)$ associated with the write pulse does not change with $\Delta t$, the profile of $p_{\tau}\left(t_{2}, t_{2}\right)$ from the read pulse is affected and exhibits a rise time that is $\sim 3$ times shorter for $\Delta t=200 \mathrm{~ns}$ than for $\Delta t=50 \mathrm{~ns}$. This prompt rise in 3(b) is consistent with the observation that stored excitation is efficiently addressed at the beginning of the read pulse for nonoverlapping write, read pulses, while the longer rise time in 3(a) results from overlapping excitation and retrieval of atoms from the state $|b\rangle$.
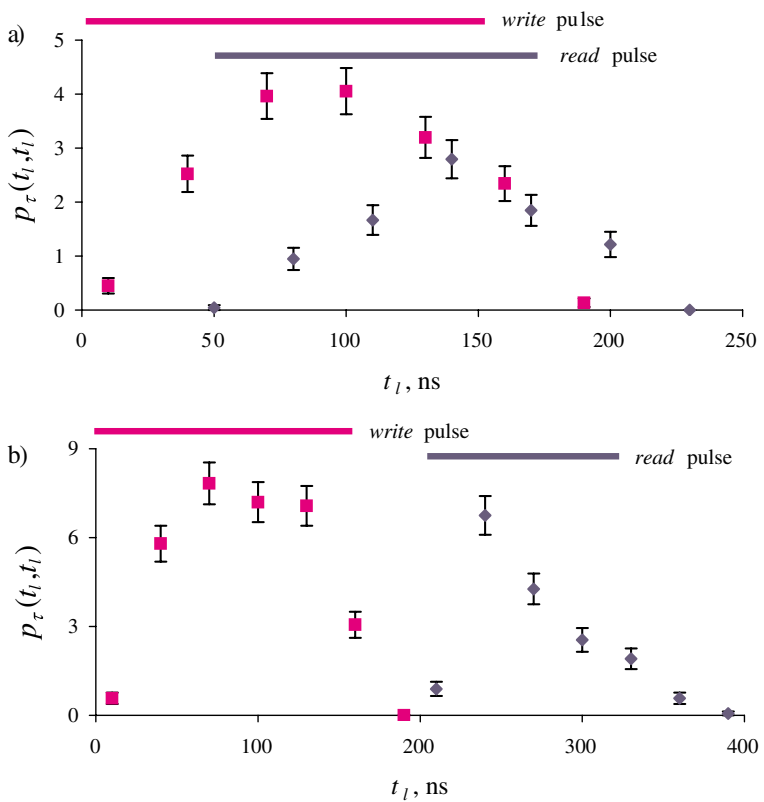

FIG. 3 (color online). Probability of joint detection scaled by $10^{8}, p_{\tau}\left(t_{1}, t_{1}\right)$ for field 1 (squares) and $p_{\tau}\left(t_{2}, t_{2}\right)$ for field 2 (diamonds) as functions of respective detection times $t_{1}$ and $t_{2}$. Bin size $\tau=30 \mathrm{~ns}$. (a) $\Delta t=50 \mathrm{~ns}$; (b) $\Delta t=200 \mathrm{~ns}$.
We employ the data in Figs. 2 and 3 together with Eq. (1) to construct $R_{\tau}\left(t_{1}, t_{2}\right)$, presented in Fig. 4 [14]. Not unexpectedly, the trends for $R_{\tau}\left(t_{1}, t_{2}\right)$ closely resemble those of $p_{\tau}\left(t_{1}, t_{2}\right)$. We observe maximal violations of CauchySchwarz inequality with $R_{\tau}^{\max }=292 \pm 57$ for $\Delta t=50 \mathrm{~ns}$ and $R_{\tau}^{\max }=202 \pm 60$ for $\Delta t=200 \mathrm{~ns}\left(R_{\tau}=198 \pm 33\right.$ in the neighboring bin), where $R_{\tau}\left(t_{1}, t_{2}\right) \leq 1$ for classical fields [7,13]. The relatively large errors in $R_{\tau}\left(t_{1}, t_{2}\right)$ arise predominantly from the uncertainties in $p_{\tau}\left(t_{1}, t_{1}\right)$ and $p_{\tau}\left(t_{2}, t_{2}\right)$ (Fig. 3) [15].

The forms for $p_{\tau}\left(t_{1}, t_{2}\right)$ and $R_{\tau}\left(t_{1}, t_{2}\right)$ for the cases $\Delta t=$ 50 and $200 \mathrm{~ns}$ imply a decoherence process operative on a time scale $\tau_{d} \sim 175 \mathrm{~ns}$. To investigate this decay, we performed a separate experiment with the delay $\Delta t$ varied $0 \leq$ $\Delta t \leq 400 \mathrm{~ns}$. For each $\Delta t$ we determine the normalized correlation function $g_{1,2}^{\tau}$ from the ratio of integrated coincidence counts to singles counts over the entire detection window (i.e., $\tau=200$ ns); see Fig. 5.

In Fig. 5, the initial growth of $g_{1,2}^{\tau}$ for small $\Delta t$ is due to the finite time required to produce sequentially photons in the $(1,2)$ fields, which is already evident in Fig. 2 and characterized by $\Delta t_{2} \simeq 60 \mathrm{~ns}$. More troublesome is the rapid decay of $g_{1,2}^{\tau}$ over times $100-200 \mathrm{~ns}$. The form for
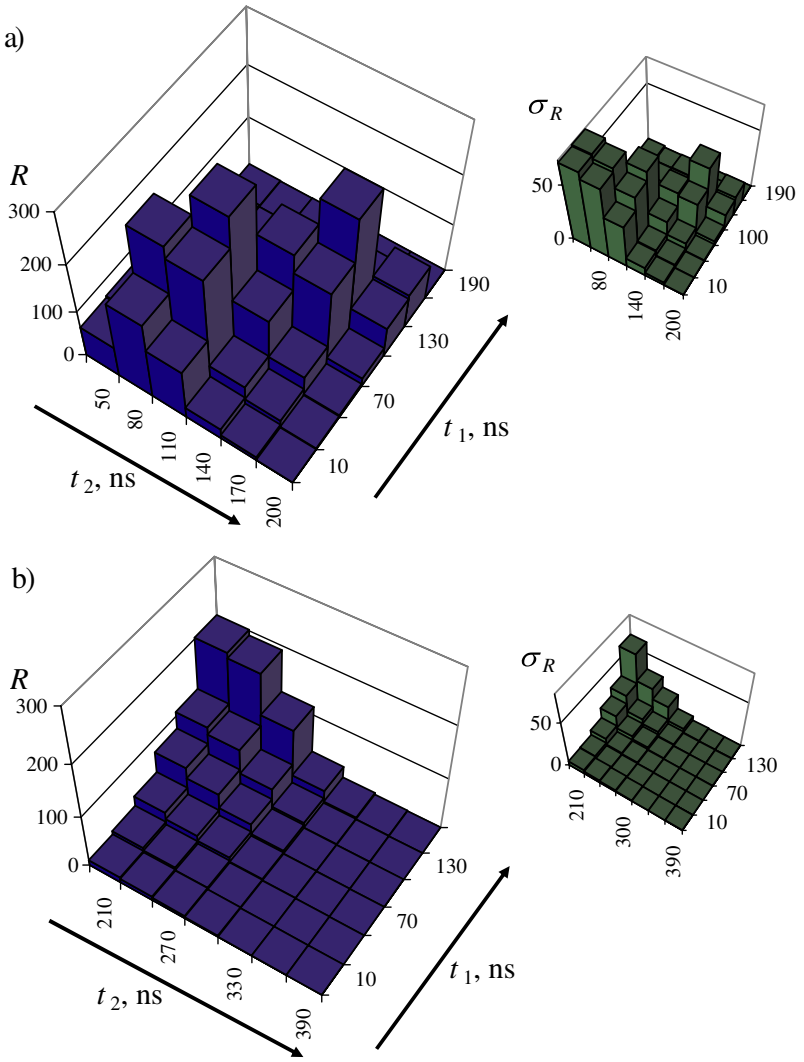

FIG. 4 (color online). The experimentally derived ratio $R_{\tau}\left(t_{1}, t_{2}\right)$ for the $(1,2)$ fields, with $R_{\tau} \leq 1$ for classical fields. The left column gives (a) $R_{\tau}\left(t_{1}, t_{2}\right)$ for $\Delta t=50 \mathrm{~ns}$ and (b) $\Delta t=200 \mathrm{~ns}$; the right column gives the associated statistical uncertainties. Bin size $\tau=30$ ns. 


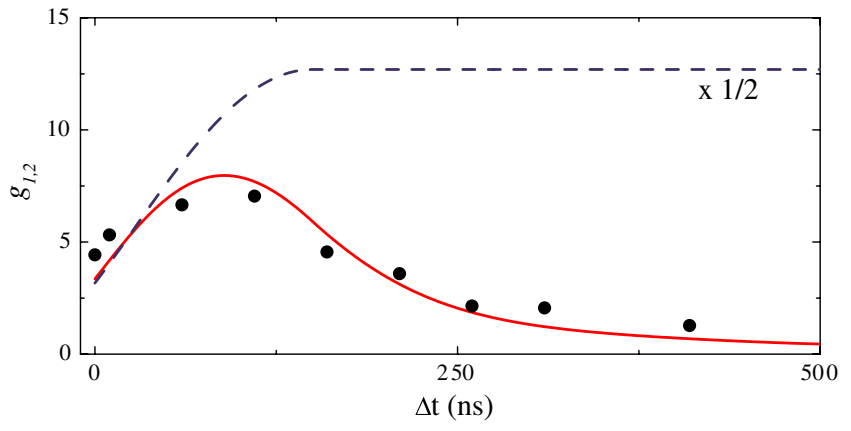

FIG. 5 (color online). Coherence time assessment. Experimentally acquired $g_{1,2}^{\tau}$ (black dots), comparison with the scaled $\tilde{p}_{1,2}(\Delta t)$ from theory (solid line), and prediction for a spin polarized $m_{F}=0$ MOT (dotted line).

$g_{1,2}^{\tau}$ versus $\Delta t$ in Fig. 5 motivates our choices $\Delta t=50$ and $200 \mathrm{~ns}$ in Figs. $2-4$. Note that $\Delta t \ll \Delta t_{12}$ would result in a substantial reduction in our $g_{1,2}^{\tau}$.

A likely cause of the quantum correlation decay is Larmor precession among the Zeeman states of the $F=$ 3,4 hyperfine levels of the $6 S_{1 / 2}$ ground level. To investigate this possibility, we have extended the treatment of Ref. [16] to include the readout process as well as the full set of Zeeman states for $F=3$, 4. The atomic ensemble is assumed to be initially unpolarized and uniformly distributed over MOT magnetic fields. With write and read pulses that approximate those used in our experiment and separated by $\Delta t$, we calculate the joint probability $p_{1,2}^{\text {th }}(\Delta t)$ to generate a pair of photons in the fields $(1,2)$. We compare the quantity $\tilde{p}_{1,2}(\Delta t) \equiv \xi p_{1,2}^{\text {th }}(\Delta t)$ to the measured $g_{1,2}^{\tau}(\Delta t)$ by way of a single overall scaling parameter $\xi$ for all $\Delta t$, as the rate of single counts in fields $(1,2)$ is measured not to depend on $\Delta t$ (to within 20\%). The result is the solid curve in Fig. 5 that adequately describes the impact of Larmor precession on our experiment. The form of $\tilde{p}_{1,2}(\Delta t)$ strongly depends upon the inhomogeneity of Zeeman splitting across the MOT, which is described by the parameter $K=\mu_{B} g_{F_{g}} L b / h$, where $L$ is the MOT diameter, $b$ is the gradient of the magnetic field for the MOT, and $g_{F_{g}}$ is the Landé factor. The curve in Fig. 5 is the theoretical result for an initially unpolarized sample with $K=1.1 \mathrm{MHz}$ as for our experiment (i.e., $L \approx 3.6 \mathrm{~mm}$ and $b \approx 8.4 \mathrm{G} / \mathrm{cm}$ ).

A remedy for this dephasing is to switch off the MOT field, and transfer the sample to a dipole-force trap. Alternatively, we are developing a scheme that should allow for long coherence times even in the presence of the quadrupole field of the MOT by utilizing only magnetic-field insensitive states. The write, read beams are circularly polarized $\left(\sigma_{ \pm}\right)$and are aligned along the $z$ axis of the MOT, which provides the quantization axis. Atoms within the approximately cylindrical volume illuminated by these beams are initially spin polarized into $F=4, m_{F}=0$ [17], where $m_{F}$ marks Zeeman sublevels. The $(1,2)$ fields are selected to be $\sigma_{ \pm}$, which results in spin excitation stored in $F=3, m_{F}=0$. The prediction of our model for this new protocol for the same experimental conditions but with an initially spin polarized sample is the dashed curve in Fig. 5, resulting in an increase of more than $3 \times$ in $g_{1,2}^{\tau}$, and significantly extending the decoherence time to more than $\tau_{d} \sim 10 \mu \mathrm{s}$.

In conclusion, we have reported the first observations of the temporal dependence of the joint probability $p_{\tau}\left(t_{1}, t_{2}\right)$ for the generation of correlated photon pairs from an atomic ensemble, which is critical for the protocol of Ref. [4]. Our measurements of $p_{\tau}\left(t_{1}, t_{2}\right)$ are an initial attempt to determine the structure of the underlying twophoton wave packet [18]. The time dependence of the ratio $R_{\tau}\left(t_{1}, t_{2}\right)$ evidences the nonclassical character of the $(1,2)$ fields with $R^{\max }=292 \pm 57 \not 1$. By contrast, previous measurements have reported $R \not 1$ only for detection events integrated over the entire durations of the write and read pulses $(R=1.84 \pm 0.06$ [7], $R=1.34 \pm 0.05$ [9], and $R=53 \pm 2$ [8]). Decoherence via Larmor precession is characterized and identified as a principal limitation of the current experiment. A new scheme for effectively eliminating this decay process is proposed and analyzed, and could be important for the experimental realization of scalable quantum networks [4] as well as for an improved source for single photons [8].

This work is supported by ARDA, the Caltech MURI Center for Quantum Networks, and the NSF; D. F. is supported by CNPQ (Brazilian Agency).

[1] C. H. Bennett et al., Phys. Rev. Lett. 70, 1895 (1993).

[2] R. Raussendorf and H. J. Briegel, Phys. Rev. Lett. 86, 5188 (2001).

[3] E. Knill, R. Laflamme, and G. J. Milburn, Nature (London) 409, 46 (2001).

[4] L.-M. Duan et al., Nature (London) 414, 413 (2001).

[5] H.-J. Briegel et al., Phys. Rev. Lett. 81, 5932 (1998).

[6] S. J. Enk, J. I. Cirac, and P. Zoller, Science 279, 205 (1998).

[7] A. Kuzmich et al., Nature (London) 423, 731 (2003).

[8] C. W. Chou et al., Phys. Rev. Lett. 92, 213601 (2004).

[9] Wei Jiang et al., Phys. Rev. A 69, 043819 (2004).

[10] M.D. Eisaman et al., quant-ph/0406093.

[11] D. N. Matsukevich and A. Kuzmich, Science 306, 663 (2004).

[12] C. H. van der Wal et al., Science 301, 196 (2003).

[13] J. F. Clauser, Phys. Rev. D 9, 853 (1974).

[14] Before applying Eq. (1), we reprocess the data for $p_{\tau}\left(t_{1}, t_{2}\right)$ in Fig. 2 for $\tau=30 \mathrm{~ns}$ bin size.

[15] To test the validity of our experiment, we verify $R\left(t_{1}^{j}, t_{2}^{k}\right)=1$ to within statistical uncertainty $\leq 4 \%$ for the $(1,2)$ fields from different trials $j \neq k$.

[16] L.-M. Duan, J. I. Cirac, and P. Zoller, Phys. Rev. A 66, 023818 (2002).

[17] Note that the beam waist for the write, read fields $w_{0}=$ $30 \mu \mathrm{m} \ll L$, where $L \approx 3.6 \mathrm{~mm}$ gives MOT diameter.

[18] K. M. Gheri et al., Fortschr. Phys. 46, 401 (1998). 